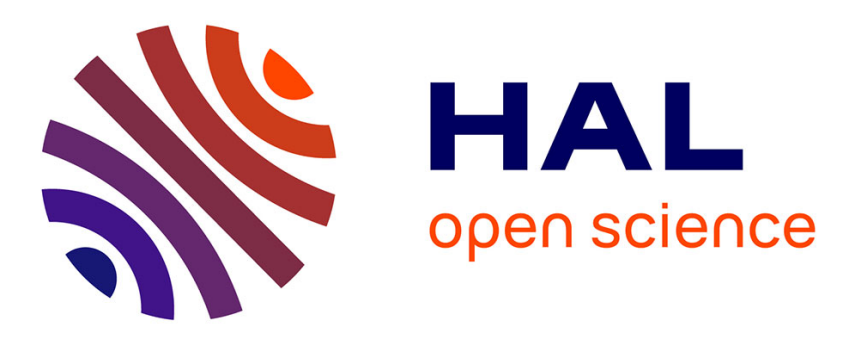

\title{
Multi-state model for dementia, institutionalization and death
}

\author{
Daniel Commenges, Pierre Joly
}

\section{To cite this version:}

Daniel Commenges, Pierre Joly. Multi-state model for dementia, institutionalization and death. Communications in Statistics - Theory and Methods, 2004, 33 (6), pp.1315-1326. inserm-00262030

\section{HAL Id: inserm-00262030 https://www.hal.inserm.fr/inserm-00262030}

Submitted on 10 Nov 2009

HAL is a multi-disciplinary open access archive for the deposit and dissemination of scientific research documents, whether they are published or not. The documents may come from teaching and research institutions in France or abroad, or from public or private research centers.
L'archive ouverte pluridisciplinaire HAL, est destinée au dépôt et à la diffusion de documents scientifiques de niveau recherche, publiés ou non, émanant des établissements d'enseignement et de recherche français ou étrangers, des laboratoires publics ou privés. 


\title{
MULTI-STATE MODEL FOR DEMENTIA, INSTITUTIONALIZATION AND DEATH
}

\section{Daniel Commenges and Pierre Joly}

\author{
Equipe de Biostatistique INSERM E03 38, ISPED, 146 rue Léo \\ Saignat, Bordeaux, 33076, France; \\ e-mail: daniel.commenges@isped.u-bordeaux2.fr
}

Article published in: Communications in Statistics A, 2004, 33, 13151326 .

ABSTRACT: We consider a multi-state model for jointly modeling dementia, institutionalization and death from data of cohort studies. Such a model could help understanding the relationships between dementia and institutionalization and also allow to make correct inferences when the initial sample of a cohort was selected as not living in institution. We consider the case where times of death and entrance in institution are known exactly while the clinical status of dementia is observed only at discrete time points. We give the likelihood in this setting and propose a penalized likelihood approach for estimating the transition intensities. A simulation study demonstrates that this non-parametric approach yields satisfactory results in this complex setting.

Key Words: dementia; illness-death model; institutionalization; interval-censoring; multi-state models; non-homogeneous Markov model.

\section{INTRODUCTION}

The illness-death model is well known and has had several applications in epidemiology. Applications and theory for inference based on right censored and left-truncated observations can be found in Andersen et al. (1). One complication arises when observations are in discrete time, producing interval-censored observations. Non-parametric maximum likelihood estimators of the transition intensities have been given by Frydman (2) for a particular observation pattern. Joly et al. (3) have 
proposed a penalized likelihood approach to this problem; Commenges (4) has given a formal proof of the likelihood for inference in an illnessdeath model with a mixed discrete-continuous observation pattern. A review of inference for multi-state models from interval-censored observations has been given by Commenges (5).

The illness-death model has been used by Joly et al. (6) to study dementia; the model was applied to the data of the Paquid cohort study, and smooth estimates of the age-specific incidence of dementia and of mortality rates of demented and not demented were given; this work was continued in Commenges et al. (7). There is another very important event that may happen to old persons: they can go into an institution. It is interesting to study the age-specific rate at which persons enter in institution; this could be done using an illness-death model interpreting the state "ill" as "institutionalized". It is interesting however to simultaneously model dementia and institutionalization using a more complex model. One of the motivation is that demented persons are (probably) much more likely to enter an institution than non-demented ones; another issue is to investigate the influence of institution on the risk of dementia. If we are interested in studying environmental factors (8) it may be important to take into account the change of this factor when the person goes in institution. Finally it may happen that the sample has been selected by a criterion related to the institutionalization status. For instance in the Paquid study the sample of the cohort was representative of subjects aged 65 years or more and living at home in a region of south-west France; moreover demented subjects at the initial visit were excluded (8). Such a selection of the sample introduces a left-truncation condition. It is relatively easy to take into account such left-truncation conditions if the event on which to condition can be represented in the model. In previous analyses using an illness-death model it was possible to take into account the condition that subjects had to be non-demented at entrance in the cohort, but it was not possible to take into account that subjects were not living in an institution.

For all these reasons we wish to jointly model dementia, institutionalization and death, taking into account the observation scheme: transitions times towards death and institution are known exactly, while the dementia status is observed only at discrete times. In this paper we develop this model, we give the likelihood from this observation pattern 
and we propose a semi-parametric approach based on penalized likelihood to estimate the transition intensities; this is done in section 2 . In section 3 we present a simulation study which shows that estimation in this complex setting-a five-state model with partially interval-censored observations-is possible with this approach.

\section{THE MODEL AND ITS LIKELIHOOD}

\subsection{THE MODEL}

We consider a five state model depicted in Figure 1; mathematically the state of a subject at time $t$ is represented by the value $X(t)$ taken by a stochastic process $X$. Subjects are in state 0 if they are not demented and living at home, in state 1 if they are demented living at home, in state 2 if they are not demented but live in institution, in state 3 if they are demented and live in institution and in state 4 if they are dead. The model is irreversible and from all states it is possible to go in the absorbing state 4 . An homogeneous model would not be realistic since it is already known that all intensities strongly depend on age. Thus we assume a non-homogeneous Markov model taking age as the basic time-scale. So the model is completely specified by the eight transition intensities $\alpha_{01}(t), \alpha_{02}(t), \alpha_{04}(t), \alpha_{13}(t), \alpha_{14}(t), \alpha_{23}(t), \alpha_{24}(t), \alpha_{34}(t)$. A semi-Markov model might also be considered because the risk of a transition to another state at time $t$ may depend on the sojourn time in the current state already elapsed at time $t$. In this paper we will not relax the Markov assumption because this would lead to sensibly more complicated computations.

\subsection{PATTERNS OF OBSERVATION}

Inference will be made on the basis of observation of the follow-up of a sample of subjects. Usually subjects are not followed-up from their birth but from a certain age which we may call age at entrance in the cohort: this is the age they have at the initial visit of the study and will be denoted $V_{0}^{i}$. Generally demented as well as institutionalized subjects are excluded from the initial sample. This produces a left-truncation that has to be taken into account in the likelihood.

We consider that, for subjects included in the cohort, time of death 
and of institutionalization are exactly observed, unless they are right censored. Right-censoring for institutionalization occurs if at the time of the analysis a subject is still alive and not institutionalized (states 0 and 1) (if a subject dies without having been institutionalized the time of institutionalization simply does not exist); for death right-censoring occurs if the subject is still alive at the time of analysis. However the clinical status of dementia is only assessed at discrete time points, which corresponds to planned visits in a cohort study: this produces intervalcensored observations. In addition there may be missing observations in the sense that a subject should have been seen at a planned visit but was not, due to refusal or other reasons. One first issue is whether the mechanism leading to these incomplete observations is ignorable. If this is the case, the likelihood can be written in terms of both transition probabilities and transition intensities and takes a relatively simple form. The mechanism leading to right-censored observations for institution or death can be considered as ignorable because for most subjects the rightcensoring variable is the date at which the analysis is done. Some subjects may be lost to follow-up and the time to entrance in institution and the time of death will be censored prior to the time of analysis. As for the observation of the clinical status, the visit times can be considered as fixed. Problems may come when these visits are missed. We shall make the assumption that anyway the mechanism leading to missing data is ignorable; the extent to which such an assumption is tenable should be discussed for a particular data set.

\subsection{The likelihood}

We give the likelihood for a generic subject of a sample. In principle all the variables, including times of observation should be indexed by $i$, the label of a particular subject; we will omit this for sake of simplicity. For obtaining the likelihood for a sample of $n$ independent subjects one must make the product of the $n$ individual likelihoods. Let $V_{0}, V_{1}, \ldots, V_{L}$ be the visit times at which the clinical status is observed. The vital and the institution statuses are observed until $C\left(C \geq V_{L}\right)$; here $V_{L}$ is the last visit time of an alive subject. Let us call $\tilde{T}_{V}$ the follow-up time that is $\tilde{T}_{V}=\min \left(T_{V}, C\right)$, where $T_{V}$ is the time of death; we observe $\tilde{T}_{V}$ and $\delta_{V}=I\left\{T_{V} \leq C\right\}$. We also observe $\delta_{I}$ which is an indicator of whether the subject has been observed institutionalized or not. Let $T_{I}$ be the 
time of institutionalization; if it is not observed we can set it to an arbitrary value since it will not appear in the likelihood. This likelihood can be written heuristically as the "probability" of the observed trajectory. Commenges (4) proved that in the case of the illness-death model this heuristic likelihood is the correct likelihood, as can be computed from Jacod's formula for multivariate counting processes (9). The likelihood is easy to write in terms of both transition intensities and transition probabilities $p_{h j}(s, t)=P(X(t)=j \mid X(s)=h)$. We shall give it for the case where there is the truncation condition $X\left(V_{0}\right)=0$ (that is for subjects selected as non-demented and living at home at entrance in the cohort).

For writing relatively simply the likelihood, we have to consider several exclusive cases. We explain how to write the first case given below; the likelihood for the other cases is written according to the same principle. Thus a subject neither seen institutionalized nor demented before the time of analysis, has been observed in state 0 from $V_{0}$ to $V_{L}$ and this happens with probability $p_{00}\left(V_{0}, V_{L}\right)$; then, since his clinical status was not observed between $V_{L}$ and $\tilde{T}_{V}$ two exclusive paths are possible: either he remained in state 0 until $\tilde{T}_{V}$ and this happens with probability $p_{00}\left(V_{L}, \tilde{T}_{V}\right)$, or he became demented during this time interval and this happens with probability $p_{01}\left(V_{L}, \tilde{T}_{V}\right)$; then according to the fact that $\tilde{T}_{V}$ was the time of death or the time of right-censoring one multiplies (or not) by the intensity of going from the state occupied just before $\tilde{T}_{V}$ to state 4 .

We first give the likelihood for a subject not demented at the time of the last visit $V_{L}$.

- If $\delta_{I}=0$ (not institutionalized)

$$
\begin{aligned}
\mathcal{L}=p_{00}\left(V_{0}, V_{L}\right) & {\left[p_{00}\left(V_{L}, \tilde{T}_{V}\right) \alpha_{04}\left(\tilde{T}_{V}\right)^{\delta_{V}}\right.} \\
& \left.+p_{01}\left(V_{L}, \tilde{T}_{V}\right) \alpha_{14}\left(\tilde{T}_{V}\right)^{\delta_{V}}\right]
\end{aligned}
$$

- If $\delta_{I}=1$ (institutionalized) 
- If $T_{I}<V_{L}$ (institutionalized before $V_{L}$ )

$$
\begin{aligned}
& \mathcal{L}=p_{00}\left(V_{0}, T_{I}\right) \alpha_{02}\left(T_{I}\right) p_{22}\left(T_{I}, V_{L}\right) \quad\left[\quad p_{22}\left(V_{L}, \tilde{T}_{V}\right) \alpha_{24}\left(\tilde{T}_{V}\right)^{\delta_{V}}\right. \\
& \left.+p_{23}\left(V_{L}, \tilde{T}_{V}\right) \alpha_{34}\left(\tilde{T}_{V}\right)^{\delta_{V}}\right]
\end{aligned}
$$

- If $T_{I}>V_{L}$ (institutionalized after $V_{L}$ )

$$
\begin{aligned}
& \mathcal{L}=p_{00}\left(V_{0}, V_{L}\right) \quad\left[p_{00}\left(V_{L}, T_{I}\right) \alpha_{02}\left(T_{I}\right) p_{22}\left(T_{I}, \tilde{T}_{V}\right) \alpha_{24}\left(\tilde{T}_{V}\right)^{\delta_{V}}\right. \\
& +p_{00}\left(V_{L}, T_{I}\right) \alpha_{02}\left(T_{I}\right) p_{23}\left(T_{I}, \tilde{T}_{V}\right) \alpha_{34}\left(\tilde{T}_{V}\right)^{\delta_{V}} \\
& \left.+p_{01}\left(V_{L}, T_{I}\right) \alpha_{13}\left(T_{I}\right) p_{33}\left(T_{I}, \tilde{T}_{V}\right) \alpha_{34}\left(\tilde{T}_{V}\right)^{\delta_{V}}\right]
\end{aligned}
$$

Then we give the likelihood for a subject first diagnosed demented at $V_{J}$.

- If $\delta_{I}=0$ (not institutionalized)

$$
\mathcal{L}=p_{00}\left(V_{0}, V_{J-1}\right) p_{01}\left(V_{J-1}, V_{J}\right) p_{11}\left(V_{J}, \tilde{T}_{V}\right) \alpha_{14}\left(\tilde{T}_{V}\right)^{\delta_{V}}
$$

- If $\delta_{I}=1$ (institutionalized)

- If $T_{I}<V_{J-1}$

$$
\mathcal{L}=p_{00}\left(V_{0}, T_{I}\right) \alpha_{02}\left(T_{I}\right) p_{22}\left(T_{I}, V_{J-1}\right) p_{23}\left(V_{J-1}, V_{J}\right) p_{33}\left(V_{J}, \tilde{T}_{V}\right) \alpha_{34}\left(\tilde{T}_{V}\right)^{\delta_{V}}
$$

- If $V_{J-1}<T_{I}<V_{J}$

$$
\begin{aligned}
\mathcal{L} & =p_{00}\left(V_{0}, V_{J-1}\right)\left[p_{01}\left(V_{J-1}, T_{I}\right) \alpha_{13}\left(T_{I}\right) p_{33}\left(T_{I}, V_{J}\right)\right. \\
& \left.+p_{00}\left(V_{J-1}, T_{I}\right) \alpha_{02}\left(T_{I}\right) p_{23}\left(T_{I}, V_{J}\right)\right] p_{33}\left(V_{J}, \tilde{T}_{V}\right) \alpha_{34}\left(\tilde{T}_{V}\right)^{\delta_{V}}
\end{aligned}
$$

- If $T_{I}>V_{J}$

$$
\mathcal{L}=p_{00}\left(V_{0}, V_{J-1}\right) p_{01}\left(V_{J-1}, V_{J}\right) p_{11}\left(V_{J}, T_{I}\right) \alpha_{13}\left(T_{I}\right) p_{33}\left(T_{I}, \tilde{T}_{V}\right) \alpha_{34}\left(\tilde{T}_{V}\right)^{\delta_{V}}
$$


For this likelihood to be useful, it must be expressed in term of the transition intensities which are the basic parameters of the model; so we must be able to express the transition probabilities in term of the transition intensities. Since our model is a Markov model the forward Kolmogorov equations give this relationship. In irreversible models these equations are easy to solve; for our model this gives:

$$
\begin{gathered}
p_{00}(s, t)=e^{-A_{01}(s, t)-A_{02}(s, t)-A_{04}(s, t)} \\
p_{11}(s, t)=e^{-A_{13}(s, t)-A_{14}(s, t)} \\
p_{22}(s, t)=e^{-A_{23}(s, t)-A_{24}(s, t)} \\
p_{33}(s, t)=e^{-A_{34}(s, t)} \\
p_{01}(s, t)=\int_{s}^{t} p_{00}(s, u) \alpha_{01}(u) p_{11}(u, t) d u \\
p_{23}(s, t)=\int_{s}^{t} p_{22}(s, u) \alpha_{23}(u) p_{33}(u, t) d u
\end{gathered}
$$

where $A_{h j}(s, t)=\int_{s}^{t} \alpha_{h j}(u) d u$.

\subsection{A semi-parametric approach to estimation}

Inference can be based on maximising the likelihood. If a parametric model is chosen, modified Newton-Raphson algorithms can be used for the maximisation. Non-parametric approaches can take two paths: one is the unconstrained non-parametric approach in the spirit of Turnbull (10) and this was developped by Frydman (2), another one uses smoothing, for instance through penalized likelihood such as in Joly and Commenges (3). In the former path the EM algorithm is attractive, in the latter the Marquardt algorithm achieves a good speed of convergence. Here we will pursue the penalized likelihood approach which is very convenient and has the advantage to yield smooth estimates of the transition intensities. However since there are eight functions to estimate in this model we propose a semi-parametric approach in order to reduce the number of functions to estimate non-parametrically. We assume that the transitions towards death are all proportional:

$$
\alpha_{14}(t)=\alpha_{04}(t) e^{\theta_{1}}
$$




$$
\begin{aligned}
& \alpha_{24}(t)=\alpha_{04}(t) e^{\theta_{2}} \\
& \alpha_{34}(t)=\alpha_{04}(t) e^{\theta_{3}}
\end{aligned}
$$

In addition we also assume proportionality of the transition intensities towards dementia:

$$
\alpha_{23}(t)=\alpha_{01}(t) e^{\theta_{4}},
$$

and proportionality of the transition intensities towards institution:

$$
\alpha_{13}(t)=\alpha_{02}(t) e^{\theta_{5}}
$$

These parametric assumptions introduce five parameters $\theta_{1}, \theta_{2}, \theta_{3}, \theta_{4}$, $\theta_{5}$; however they reduce to three the number of functions on which no parametric assumptions are made. The numerical complexity is thus no more than for the three-state illness-death model treated by Joly et al. (6). The penalized likelihood is:

$$
p l(\alpha(.), \beta, D)=l(\alpha(.), \theta, D)-\sum_{j=1,2,4} \kappa_{0 j} \int_{0}^{\infty}\left[\alpha_{0 j}^{\prime \prime}(u)\right]^{2} d u
$$

where $l$ is the loglikelihood, $\alpha($.$) is the matrix of baseline hazard func-$ tions, $\theta$ is the vector of proportionality parameters, $D$ represents the data. The penalty term excludes that discrete or unsmooth functions maximise $p l(\alpha(),. \beta, D)$ and its weight is tuned by the smoothing coefficients $\kappa_{01}$, $\kappa_{02}, \kappa_{04}$.

The solution of the penalized likelihood is approximated using a basis of splines. We use cubic M-splines which are variants of B-splines (11). For $j=1,2,4$ we consider transition intensities generated by linear combinations of M-splines; if we use the same basis of splines for the three intensities we can write: $\tilde{\alpha}_{0 j}()=.\tilde{\boldsymbol{\gamma}}_{j} \boldsymbol{M}($.$) , where \boldsymbol{M}()=$. $\left(M_{1}(.), \ldots, M_{m}(.)\right)^{T}$ is a basis of M-splines and $\tilde{\gamma}_{j}=\left(\tilde{\gamma}_{j 1}, \ldots, \tilde{\gamma}_{j m}\right)$ are the parameters. We may also use distinct bases of splines for the different transition intensities, possibly with a different number of splines in each basis. The positivity constraint for $\tilde{\alpha}_{0 j}($.$) is fulfilled by constraining the$ coefficients $\tilde{\gamma}$ to be positive. The approximation $\tilde{\alpha}$ of $\hat{\alpha}$ is the set of functions belonging to the space generated by the basis of splines, which maximizes $p l\left(\alpha_{01}, \alpha_{12}, \alpha_{02}, \theta\right)$. From a numerical point of view we have to maximise over the parameters of the splines $\tilde{\gamma}_{j}, j=1,2,4$ plus the five 
proportionality parameters. In principle explanatory variables can also been added, as has been done in Commenges et al. (7).

The three smoothing parameters $\kappa_{01}, \kappa_{02}, \kappa_{04}$ can be chosen by maximising a likelihood crossvalidation criterion as in Joly et al. (6). The efficiency of the global procedure has not been analytically studied but several simulations showed a very satisfactory behaviour. We may think that the efficiency relative to the good parametric model is high as long as the functions to estimate are really smooth.

\section{A SIMULATION STUDY}

A simulation study was performed to demonstrate the feasability of the proposed semi-parametric approach to the rather complex setting of this five-state model with partially interval-censored observations. We generated three samples of sizes 500, 2000 and 4000 observations according to this model and choosing Weibull intensities for the baseline intensities. Precisely the three simulated baseline intensities were of the form:

$$
\alpha(t)=\lambda p(\lambda t)^{p-1}
$$

and the parameters $(\lambda, p)$ were $(0.05,2.4),(0.08,2.6)$ and $(0.06,2.5)$ for $\alpha_{01}, \alpha_{02}$ and $\alpha_{04}$ respectively. The other transitions were obtained following the proportionality assumptions of the previous section with $\theta_{1}=0.8$, $\theta_{2}=0.2, \theta_{3}=1, \theta_{4}=1$ and $\theta_{5}=0.6$. These values corresponded to proportionality constant equal to $2.22,1.22,2.72,2.72$ and 1.82. For instance the mortality rate of demented subjects, that is the transition intensity from state 1 to state 4 is: $\alpha_{14}(t)=\alpha_{04}(t) e^{\theta_{1}}=2.22 \lambda p(\lambda t)^{p-1}$ with the values $(\lambda=0.06, p=2.5)$.

All the generated processes started from state 0 at time zero. For each subject, a left-truncation variable with uniform distribution on $[0,5]$ was generated: observations were included only if no transition had occured previous to the value of the truncation variable. For each subject, a rightcensoring variable was generated from a uniform distribution on $[2,52]$. Times of transition towards institution or death was exactly observed unless right-censored. The clinical status of dementia was observed at dicrete visit times generated as $V_{j+1}=V_{j}+U_{j+1}, j=0, \ldots, L-1$, where the $U_{j}$ were independently uniformly distributed on $[1,4]$ and $V_{L}<\tilde{T}_{V}$. Thus the mean duration between two visits was 2.5. We added 65 to all 
the generated times so that these times look like ages expressed in year, at which the event considered may be observed in cohort of persons aged more than 65 .

The three baseline functions were approximated using a basis of splines with 7 knots for each. The penalized likelihood was maximised on the 32 parameters (27 spline parameters plus five proportionality parameters) using a Marquardt algorithm (12) (a robust version of the NewtonRaphson algorithm), implemented through a Fortran program; for fixed smoothing coefficients, the algorithm converged in about 12 iterations. The likelihood crossvalidation criterion was maximised over the three smoothing coefficients $\kappa_{0 j}$ using a simple grid method.

The estimates for the three baseline intensities for $n=2000$ are compared to the simulated ones in Figure 2. The estimates of the proportionality constants are given in Table 1 for the three simulations with $n=500,2000,4000$. Confidence intervals are also given for the proportionality constants: these are based on the hessian of the penalized likelihood (that is treating penalized likelihood as if it was a likelihood), an approach proposed by O'Sullivan (13) and others. This is justified by a Bayesian argument identifying the penalized likelihood to an a posteriori distribution. Globally the results appear very satisfactory since the estimated curves are near the simulated ones and the estimated constants are well inside the computed confidence intervals (unfortunately the length of the procedure excludes evaluating the coverage of these confidence intervals by replicating this simulation a large number of times). Moreover one can see that the estimated width of the confidence intervals are shorter for larger sample sizes and that their ratios are roughly proportional to the inverse of the square root of the sample sizes. For instance, for $\exp \theta_{1}$ the ratios of the lengths of the confidence intervals obtained for $n=2000$ and $n=4000$ to that obtained for $n=500$ are 0.43 and 0.34 respectively, which is close to what is expected (0.5 and 0.34 ) for the classical convergence speed in $n^{1 / 2}$.

In further work we intend to apply this method to the data of the Paquid study which should yield interesting epidemiologic results at both a descriptive level and analytic one, by giving insight on the relationship between dementia and intitutionalization.

\section{BIBLIOGRAPHY}


(1) Andersen, P.K.; Borgan Ø.; Gill R.D.; Keiding N. Statistical Models Based on Counting Processes. New-York: Springer-Verlag, 1993

(2) Frydman, H. Non-parametric estimation of a Markov "illness-death model" process from interval-censored observations, with application to diabetes survival data. Biometrika, 1995, 82, 773-789.

(3) Joly, P.; Commenges, D. A penalized likelihood approach for a progressive three-state model with censored and truncated data: Application to AIDS. Biometrics, 1999, 55, 887-890.

(4) Commenges, D. Likelihood for interval-censored observations from multi-state models. Statistics and Operational Research Transactions, 2003, in press.

(5) Commenges, D. Inference for multi-state models from interval-censored data. Statistical Methods in Medical Research, 2002, 11, 167-182.

(6) Joly, P.; Commenges, D.; Helmer, C.; Letenneur, L. A penalized likelihood appproach for an illness-death model with interval-censored data: application to age-specific incidence of dementia. Biostatistics, 2002, 3, 433- 443 .

(7) Commenges, D.; Joly, P. ;Letenneur, L.; Dartigues, JF. Incidence and prevalence of Alzheimer's disease or dementia using an Illness-death model. Statistics in Medicine, 2004, 23, 199-210.

(8) Rondeau, V.; Commenges, D.; Jacqmin-Gadda, H.; Dartigues, JF. Relationship between aluminium and silica concentrations in drinking water and Alzheimer's disease: an 8-year follow-up study. Am J Epidemiol, 2000, 152, 59-66.

(9) Jacod, J. Multivariate point processes: predictable projection; RadonNikodym derivative, representation of martingales. Z. Wahrsheinlichkeitsth., 1975, 31, 235-253.

(10) Turnbull, B. W. The empirical distribution function with arbitrarily grouped, censored and truncated data. Journal of the Royal Statistical Society, Series B, 1976, 38, 290-5.

(11) Joly, P.; Commenges, D.; Letenneur, L. A penalized likelihood ap- 
proach for arbitrarily censored and truncated data: application to agespecific incidence of dementia. Biometrics, 1998, 54, 185-194.

(12) Marquardt, D. An algorithm for least-squares estimation of nonlinear parameters. SIAM Journal of Applied Mathematics, 11, 431-441.

(13) O'Sullivan F. (1988) Fast computation of fully automated log-density and log-hazard estimators. SIAM Journal on Scientific and Statistical Computing, 1963, 9, 363-379. 
Table 1: Simulated and estimated proportionality constants in the semiparametric model

\begin{tabular}{|c|c|c|c|c|c|c|c|}
\hline \multirow[b]{2}{*}{$\mathrm{j}$} & \multirow[b]{2}{*}{$\exp \left(\theta_{j}\right)$} & \multicolumn{2}{|c|}{$n=500$} & \multicolumn{2}{|c|}{$n=2000$} & \multicolumn{2}{|c|}{$n=4000$} \\
\hline & & $\exp \left(\hat{\theta}_{j}\right)$ & $95 \%$ CI & $\exp \left(\hat{\theta}_{j}\right)$ & $95 \%$ CI & $\exp \left(\hat{\theta}_{j}\right)$ & $95 \%$ CI \\
\hline 1 & 2.22 & 2.53 & {$[1.40,4.59]$} & 1.99 & {$[1.42,2.78]$} & 2.41 & {$[1.93,3.02]$} \\
\hline 2 & 1.22 & 1.14 & {$[0.81,1.62]$} & 1.12 & {$[0.96,1.32]$} & 1.24 & {$[1.11,1.38]$} \\
\hline 3 & 2.72 & 2.85 & {$[2.03,4.01]$} & 2.38 & {$[2.01,2.81]$} & 2.50 & {$[2.22,2.82]$} \\
\hline 4 & 2.72 & 2.81 & {$[1.98,3.97]$} & 2.55 & {$[2.12,3.08]$} & 2.85 & {$[2.51,3.24]$} \\
\hline 5 & 1.82 & 2.20 & {$[1.46,3.31]$} & 1.94 & {$[1.53,2.47]$} & 1.60 & {$[1.35,1.90]$} \\
\hline
\end{tabular}

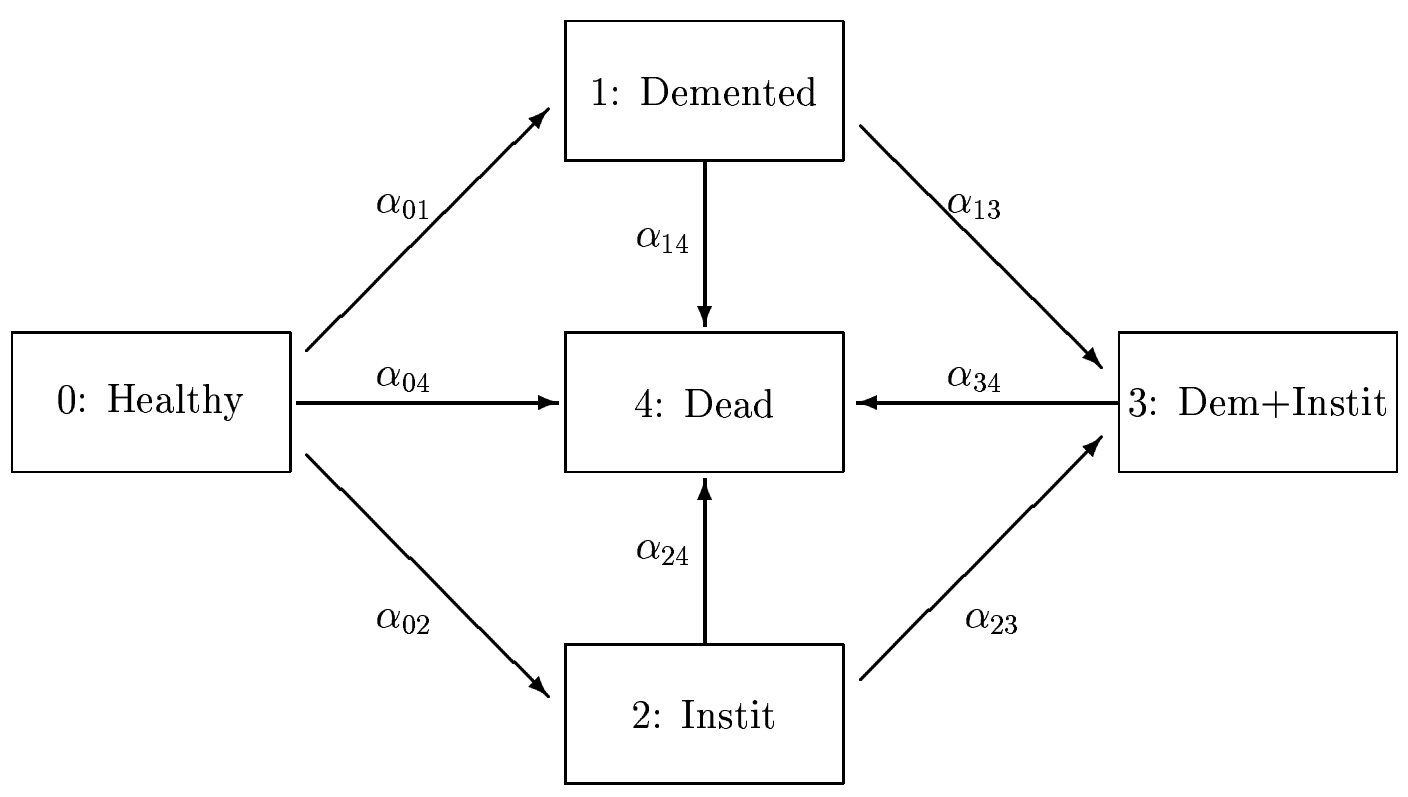

Figure 1: The five-state model for dementia, institutionalization and death. 


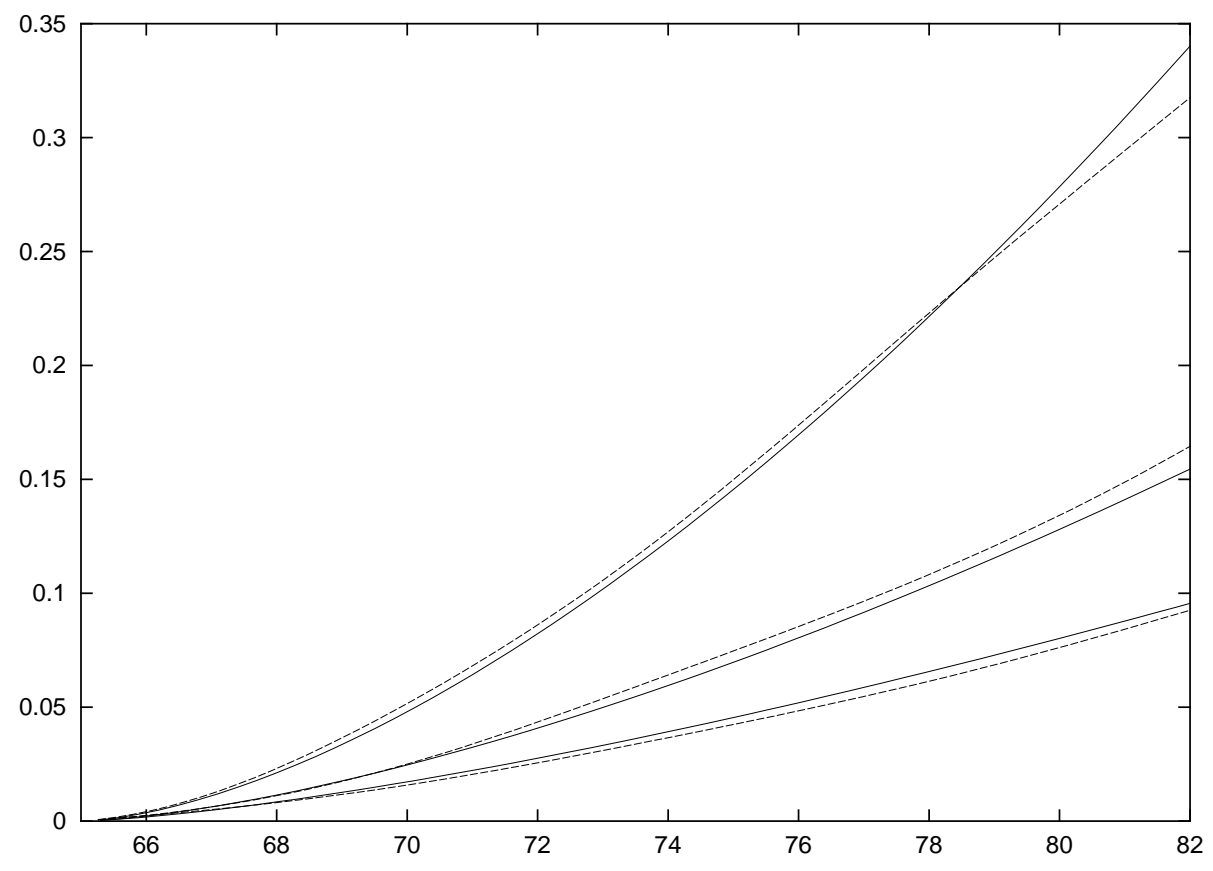

Figure 2: Comparison of the three baseline estimated (dotted lines) and simulated (full lines) intensities: upper: $\alpha_{02}$; middle: $\alpha_{04}$; lower: $\alpha_{01}$; sample size: $n=2000$. 